\section{Submucosal carcinoma of the gastroesophageal junction diagnosed after peroral endoscopic myotomy}
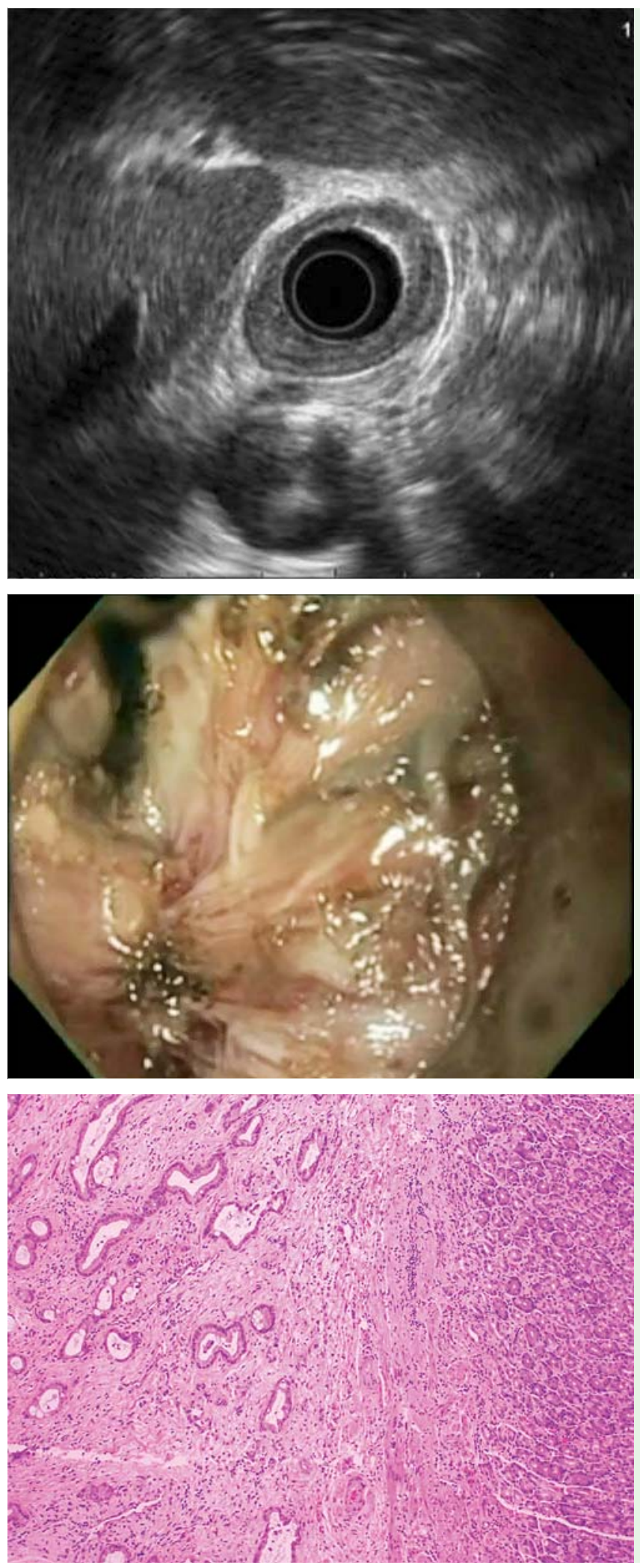

Fig. 1 Endoscopic ultrasound (EUS) in a 62 year old man with type II achalasia showing a circumferential thickening at the area of the gastroesophageal junction (GEJ) but no mass lesions.

Fig. 2 Image taken during peroral endoscopic myotomy (POEM) showing the scarred and infiltrating tissue that was found during submucosal dissection at the level of the abnormally thickened and indurated gastroesophageal junction (GEJ).

Fig. 3 Final histology following total gastrectomy showing normal looking mucosa on the right of the image and a submucosal tumor not infiltrating beyond the muscularis mucosa on the left of the image (magnification $\times 100$ ).
Gastroesophageal junction (GEJ) malignancies are found in $4.7 \%$ of patients who fulfil the manometric criteria for achalasia [1]. Such malignancies may manifest as pseudoachalasia because of submucosal infiltration and secondary impairment of the inhibitory neurons of the esophageal myenteric plexus, therefore mimicking the manometric pattern of achalasia [2]. Despite endoscopic biopsies, false-negative rates of $25 \%$ may mask this cause of pseudoachalasia [3].

A 62 year old man with no history of Barrett's esophagus presented with a 4-month history of dysphagia, vomiting, and weight loss. His initial esophagogastroduodenoscopy (EGD) showed a dilated esophagus and tight GEJ; multiple biopsies were negative. A computed tomography (CT) scan of the thorax and abdomen showed a bulky GEJ with no definite mass lesion. Endoscopic ultrasound (EUS) also showed a circumferential thickening in the area of the GEJ, but no masses suggestive of malignancy or suspicious lymph nodes were seen ( $\bullet$ Fig. 1 ). High resolution manometry suggested type II achalasia.

As malignancy had been excluded as a cause of the tight GEJ, the patient underwent peroral endoscopic myotomy (POEM). During creation of the submucosal tunnel, thickening and fibrosis of the muscularis propria was encountered near the GEJ ( $\bullet$ Fig. 2); however, intraoperative frozen sections were normal. The remainder of the POEM proceeded uneventfully, the myotomy being performed with a Triangle Tip knife (Olympus) and the tunnel entry being closed with clips ( Video1). Multiple biopsies of the mucosa and muscularis propria showed cytologic atypia of unknown significance but were negative for malignancy.

The patient was monitored closely in the clinic and his symptoms recurred within a month. Another EGD showed a GEJ stricture $38 \mathrm{~cm}$ from the incisors. Biopsies were again indeterminate. A repeat CT scan confirmed a 3.6-cm concentric mural thickening at the GEJ. Surgery was therefore advised. Intraoperatively, a localized tumor was found at the GEJ. A total gastrectomy with D2 lymphadenectomy was performed. Final histology revealed a moderately differentiated submucosal adenocarcinoma of the GEJ with no involvement of the mucosa ( $\bullet$ Fig.3).

After receiving chemotherapy, the patient remained free of symptoms and disease 1 year postoperatively. 


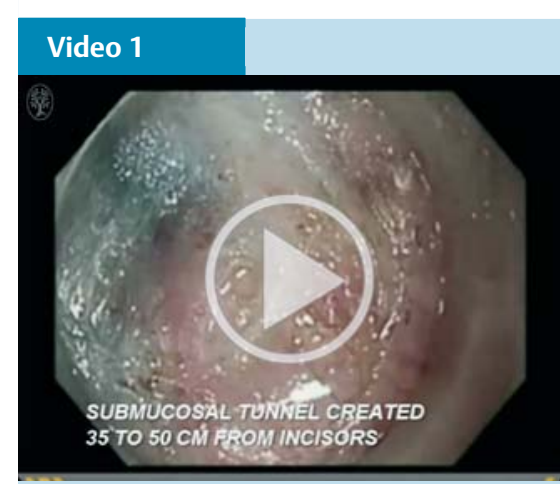

Views of the pre-procedure imaging; the stages of the peroral endoscopic myotomy (POEM) procedure showing the abnormal tissue found at the gastroesophageal junction (GEJ) during tunnel dissection and closure of the tunnel entry with clips; the macroscopic and microscopic appearances of the specimen subsequently resected at gastrectomy (CT, computed tomography; EUS, endoscopic ultrasound; 12'OC, 12 o'clock position; TTknife, Triangle Tip knife).
This case highlights the rare occurrence of a submucosal GEJ adenocarcinoma that was not identified on either pre-POEM endoscopy with mucosal biopsies or on EUS in a patient with symptoms and signs suggestive of achalasia. This may have been due to a submucosal tumor and sampling error. After performing POEM, clinicians should maintain a degree of suspicion for malignancy if a patient's symptoms fail to resolve.

\section{Endoscopy_UCTN_Code_CPL_1AH_2AJ}

Competing interests: None

\section{Dexter Yak Seng Chan', Dmitrii Dolgunov ${ }^{1}$, Victor Kwan Min Lee ${ }^{2}$, Philip Wai Yan Chiư ${ }^{3}$, Jimmy Bok \\ Yan So ${ }^{1}$}

${ }^{1}$ Department of Surgery, National University Health System, Singapore, Singapore

2 Department of Pathology, National University Health System, Singapore, Singapore

${ }^{3}$ Department of Surgery, The Chinese University of Hong Kong, Hong Kong, China

\section{References}

1 Campo SM, Zullo A, Scandavini CM et al. Pseudoachalasia: a peculiar case report and review of the literature. World J Gastrointest Endosc 2013; 5: 450-454

2 Rozman RW, Achkar E. Features distinguishing secondary achalasia from primary achalasia. Am J Gastroenterol 1990; 85: 1327 1330

3 Woodfield CA, Levine MS, Rubesin SE et al. Diagnosis of primary versus secondary achalasia: reassessment of clinical and radiographic criteria. Am J Roentgenol 2000; 175: 727 731

\section{Bibliography}

DOI http://dx.doi.org/

10.1055/s-0034-1393591

Endoscopy 2015; 47: E623-E624

(c) Georg Thieme Verlag KG

Stuttgart · New York

ISSN 0013-726X

\section{Corresponding author} Jimmy Bok Yan So, MD

Department of Surgery National University Health System

1 E Kent Ridge Road

NUHS Tower Block, Level 8

Singapore 119228

Singapore

jimmy_so@nuhs.edu.sg 